7th International Workshop on Astronomy and

Relativistic Astrophysics (IWARA 2016)

International Journal of Modern Physics: Conference Series

Vol. 45 (2017) 1702001 (1 page)

(C) World Scientific Publishing Company

DOI: $10.1142 /$ S2010194517020013

\title{
Peer Review Statement
}

\author{
Published 15 August 2017
}

\begin{abstract}
All articles in this volume have been subjected to peer review administered by the proceedings editors and have been revised in accordance with comments of the expert referees.
\end{abstract}

